\title{
Mecánicas emocionales y proyectivas en las estrategias de activación patrimonial y museística
}

\section{Projective and Emotional Mechanics in the Strategies of Museum and Heritage Activation}

\author{
Iñaki DÍAZ BALERDI \\ Universidad del País Vasco / Euskal Herriko Unibertsitatea \\ Facultad de Letras. Dto. Historia del Arte \\ Av. Universidad, 5 \\ 01006 Vitoria-Gasteiz \\ i.diazbalerdi@ehu.es
}

Recibido: 1-04-2014

Aceptado: 15-09-2014

\begin{abstract}
Resumen
Las mecánicas patrimoniales se han basado tradicionalmente en factores racionales, marginando aquellos de carácter más emotivo o relacional. Además, en los últimos tiempos el sesgo economicista las ha obligado a buscar la rentabilidad y participar en el juego de la oferta y la demanda, con unos resultados muy poco esperanzadores.

Cambiar ese estado de cosas pasa por cambiar radicalmente las estrategias puestas en marcha, basarse en la interlocución, conocer las relaciones entre el patrimonio y los colectivos sociales y, sobre todo, cambiar nuestra manera de pensar en el patrimonio.
\end{abstract}

Palabras Clave: Patrimonio. Turismo cultural. Emotividad. Interlocución. Colectivos sociales.

\begin{abstract}
The patrimonial mechanics has been based traditionally on rational factors, isolating those of more emotive or relational character. In addition, in the last times, The economic bias has forced them to seek for the profitability and to take part in the game of the offer and the demand, with poor results.

To change this state of things means to change radically the strategies, to be based on the dialogue, to know the relations between the heritage and the social groups and, especially, to change our way of thinking about the heritage.
\end{abstract}

KEY worDs: Heritage. Cultural tourism. Emotivity factors. Dialog. Social groups. 
La lógica de un museo parte siempre de la oferta, no de la demanda (Donaire 2012: 36).

Miles de museos pueblan el mundo. Museos de todo tipo, tamaño y condición. Si añadimos arquitecturas singulares, lugares con transcendencia histórica o social, monumentos, parques naturales y centros de interpretación podríamos inferir que vivimos inmersos en escenarios entreverados -si ellos mismos no lo son- de enclaves patrimoniales, de manera que, a priori, resultaría difícil sustraerse a su influencia o, cuando menos, a su presencia.

Sin embargo ¿cuántos habitantes de ese mundo engalanado de acervos patrimoniales se sienten afectados por los productos y las posibilidades que brindan las instituciones garantes de la conservación y socialización del patrimonio? ¿Cuántos acuden con cierta regularidad a contactar con esas plasmaciones objetuales o intangibles de la memoria, de la historia, del conocimiento, del genio creativo? ¿Cuántos participan de manera activa en las estrategias de salvaguarda patrimonial?

Al margen de que sería necesario aquilatar el alcance de varios términos utilizados en el párrafo anterior, la respuesta a todas las preguntas $-\mathrm{y}$ se podrían plantear muchas más- es la misma. Pocos. Tan pocos que, en las últimas décadas, una especie de mantra entrevera el día a día de esos enclaves patrimoniales, proclamando la necesidad de que el número de sus visitantes aumente y de que la socialización antes mencionada se proyecte urbi et orbe.

Antes, en los buenos ( $i$ ?) viejos tiempos, se suponía que el hecho de conservar y de facilitar la contemplación de parte de lo conservado era suficiente para que museos y otras instituciones cumplieran con su misión y objetivos, mientras el factor "visitante" tan solo era algo tangencial, residual. Pero las cosas cambian. Y de qué manera.

Sobre todo desde que algunos centros se convirtieron en destinos anhelados de peregrinación cultural, recabaron para sí una atención mediática inédita y demostraron que su existencia no constituía tan solo un ornato de buen gusto o una manifestación de las glorias del lugar, sino que, además, se podían revelar como factores importantes en asuntos tan dispares como economía, turismo, bienestar, etc., etc. Es decir, desde que demostraron que, aun siendo por lo general deficitarios en términos puramente contables, también podían producir réditos directos o indirectos que contribuían a la mejora de los balances financieros del territorio donde se ubicaban.
Las consecuencias de esos cambios no se hicieron esperar. Por un lado, se produjo algo parecido a un efecto llamada que conduciría a una suerte de desbordado incremento del consumo cultural y, a su vez, a la multiplicación de nuevos centros dedicados a tareas patrimoniales. Y, por otro, se asumió que si algunas de esas instituciones habían demostrado su viabilidad -más allá de su mera pertinencia-, en adelante, esa viabilidad sería una de las exigencias -o de los deseos- a la hora de proponer nuevas iniciativas, las cuales deberían demostrar, aunque fuera de manera proyectiva, su capacidad para crear empleo, captar turismo, configurarse como marca e imagen y, en definitiva, generar beneficios.

Con estas premisas no resulta raro que el mantra relativo a la deseable multiplicación de visitantes se asentara y difundiera. Otra cosa es que se consiguieran los resultados esperados, que evidentemente no fue así. O, en todo caso, fue así en un reducido número de instituciones, mientras que la inmensa mayoría seguía, sigue y seguirá moviéndose en parámetros solo ligeramente beneficiados por esa nueva modalidad de consumo y matizados por los altibajos en los niveles de bienestar general.

La crisis, que no acaba de acabar, se ha convertido en un espejo que nos devuelve una imagen diferente de lo que estábamos acostumbrados a ver -o a imaginar- respecto a la relación que se establece entre los individuos -y los colectivos sociales- y el patrimonio. En principio, porque los índices de asistencia a museos y lugares patrimoniales bajan o, en todo caso, se mantienen en algunos casos gracias a operaciones de fuerte impacto mediático y social (la exposición, en 2013, sobre Dalí en el Reina Sofía sería un buen ejemplo). En segundo lugar, por las dificultades económicas de la mayoría de instituciones que trabajan con bienes patrimoniales, algunas de las cuales, por cierto, ya se han visto condenadas a cerrar. En tercer lugar, y por no alargar la lista, porque desde distintas instancias se comienza a alertar sobre lo equivocado de aquel optimismo generalizado que atribuía propiedades miríficas al patrimonio en un sistema económico antagónico -en cuanto a principios y metodologías- con algunos de los aspectos singulares del patrimonio como bien de uso común.

Si esto es así -y ojalá no lo fuera y todo marchara miel sobre hojuelas- podríamos preguntarnos acerca del por qué de la inquietante situación en que nos encontramos. Y también acerca de los rumbos que habría que rectificar para mejorar la 
operatividad del patrimonio y de sus relaciones con la sociedad.

Es lo que haremos en las páginas que siguen, conscientes de que las fórmulas mágicas no existen, de que lo que se lea a continuación solo es una propuesta de discusión susceptible de ser enriquecida mediante distintos aportes o, en todo caso, para qué negarlo, rebatida en su fondo y forma. En cualquier circunstancia, será mejor discutir que movernos, como lo hemos hecho durante mucho tiempo, por inercias y presupuestos -conceptuales y pragmáticos- que no concordaban con una realidad siempre más tozuda que cualquier actitud biempensante y poco propensa a someterse al debate, la confrontación o la crítica.

Todo pasa y todo queda, pero lo nuestro es pasar, pasar haciendo caminos, caminos sobre la mar,

Los versos de Machado, además de constituir un buen hilo conductor para nuestra argumentación, serían perfectamente aplicables al mundo de los museos y de los bienes patrimoniales. Lo serían por la permanencia de los mismos y también por los incesantes cambios en sus rumbos y mecánicas, hasta el punto de que a veces se nos antojan volubles, aleatorios e impredecibles. Lo que ayer era válido hoy lo ponemos en cuestión. $\mathrm{Y}$ lo que ahora tenemos por cierto ¿quién nos dice que no será cuestionado pasado mañana?

Para empezar con nuestro análisis, deberíamos recordar que los museos han dejado de ser los únicos entes autorizados en la gestión patrimonial. Hasta hace unos años la distinción, por ejemplo, entre museo y centro de interpretación parecía clara -al fin y al cabo los primeros se definían por sus colecciones-, pero hoy en día las tipologías no son tan evidentes. Lo mismo pasa en cualquier otro campo, desde la culinaria al arte: tanto sus definiciones conceptuales como los formatos en que se expresan o materializan rompen sin cesar moldes canónicos, diluyen fronteras y compartimentaciones estancas y dan lugar a la aparición de nuevas tipologías híbridas, maleables, evolutivas.

Por otro lado, deberíamos partir de la idea de que el patrimonio no es algo preexistente o, en todo caso que, aunque estrictu sensu lo sea, adquiere cartas de naturaleza en el momento que lo investimos con los atributos de dicho concepto. Es decir, el patrimonio se construye, y esa cons- trucción no solo se fundamenta en razonamientos científicos sino que se reviste de implicaciones ideológicas: la construcción patrimonial es un hecho eminentemente político (Karp 1991), por lo que la codificación patrimonial siempre será discutible (Clifford 1985), al ser la consecuencia de la elección de una opción entre varias posibles.

La progresiva complejidad del concepto "patrimonio" y la elasticidad tipológica de sus escenificaciones producen, por un lado, una ampliación o enriquecimiento de las posibilidades de mirada y de acción, pero también ciertas zozobras, al no ser el terreno que pisamos tan estable y previsible como antaño: una de las que ha alcanzado últimamente un cariz casi obsesivo en lo que atañe a todo lo relacionado con la gestión y las finanzas, hasta el punto de convertirse en los tiempos que corren en los verdaderos ejes en torno a los cuales gira cualquier actividad. El sesgo marcadamente economicista del pensamiento neoliberal -que es el que se nos impone desde las más altas instancias del poder- implica que las instituciones patrimoniales deban jugar, como cualquier otra, en el juego de la oferta y la demanda con un objetivo doble: satisfacer a patrones y fidelizar la clientela.

También en la misma lógica se externalizan actividades, lo cual no es sino una privatización parcial o encubierta de un servicio en principio público, o se recurre sistemáticamente a campañas de marketing semejantes a las que podamos encontrar en otros sectores económicos. Sin olvidar, claro está, el protagonismo creciente de la iniciativa privada como agente activo, tanto como poseedora, pongamos por caso, de colecciones propias como por la adquisición o usufructo de enclaves patrimoniales reconvertidos para un uso que aúna el valor del bien y la nueva función a la que se le destina (hoteles, etc.). Aquí también la hibridación y el desdibujamiento de los límites tipológicos se imponen.

Quizá el campo donde mejor se visualiza este cambio teñido de economicismo es en eso que se ha dado en llamar turismo cultural, locución que ha tenido una gran acepción, sobre todo para designar a ese tipo de visitas o desplazamientos en los que "lo cultural" ocupa un lugar destacado: puede tratarse de conocer iglesias, museos y demás especímenes del patrimonio material, pero también englobar manifestaciones de lo intangible, como fiestas, tradiciones, gastronomía, etc.

El binomio turismo-cultura fue visto como tabla de salvación y promesa de innumerables beneficios (Prats 2003), aunque el asunto, en tér- 
minos generales y con las obligadas excepciones, no cumplió con las expectativas de tanto estratega hechizado por aquel canto de sirenas y, a día de hoy, los resultados de tales aventuras son muy discutibles (un minucioso y bien documentado acercamiento al tema lo podemos encontrar en Arrieta 2012). Lo que habría que preguntarse es si se fomentó un turismo al servicio del patrimonio -pues ahí podía materializarse una efectiva vía de socialización, a la vez que parte de los rendimientos obtenidos podrían ser reinvertidos en su mejor conservación y difusión- o si el patrimonio fue visto como un reclamo de buen tono para un apetecible negocio.

Solo hay que pensar en cuántos de los cientos de enclaves patrimoniales inaugurados a partir de los años 80 del siglo pasado en nuestro país se han convertido en rutilantes estrellas del firmamento patrimonial. Pocos, otra vez. La mayoría ocupa más bien un lugar bastante sombrío, por no decir inexistente, en el imaginario colectivo, lo cual no quiere decir, por supuesto, que habría que cerrarlos todos. No, pero habría que recordar -y aplicar a nuestro contexto- las palabras de Keneth Hudson (1989) cuando alertaba sobre la proliferación de museos innecesarios, y constatar que muchos de ellos son producto de una fiebre pasajera que apenas sopesaba la viabilidad real de proyectos que, una vez inaugurados -y acabados los fastos, las fotos y el rédito en términos de imagen y presencia en los medios de comunicación-, pasaban a engrosar el abultado número de equipamientos marginales, costosos y con escasa proyección social.

Pero tampoco podemos olvidar que por debajo de -o paralelo a- la urgencia, improvisación, falta de planificación estratégica y coordinación, e, incluso, cierto papanatismo mimetizador en las ensoñaciones modernizadoras de tantos y tantos políticos y gestores culturales, también existía, o podía existir, una auténtica necesidad de dotar a nuestra geografía de unos equipamientos que garantizaran la conservación y difusión de numerosos bienes patrimoniales que hasta entonces no habían recibido las atenciones necesarias. $\mathrm{Ni}$ pasar por alto la existencia de individuos, grupos o asociaciones empeñados contra viento y marea en esas tareas en las que las administraciones no ponían excesivo interés, sobre todo cuando el asunto a conservar y dignificar no venía aureolado de los oropeles que otorga la presencia mediática.

Probablemente aquellas estrategias -si es que así se les puede denominar- fracasaron, pero lo hicieron porque estaban animadas en el fondo por el convencimiento de que la apertura de un museo, de un centro de interpretación, etc., iba a producir de manera automática una multiplicación del número de turistas interesados en conocer las nuevas maravillas puestas a su disposición, sin darse cuenta de que eso era muy improbable -como el tiempo se encargó de demostrar- y de que, al no discriminar lo que se estaba patrimonializando, esas maravillas -cuando existían- o no eran tales, al menos para ojos ajenos, o se repetían, sin aportar nada novedoso, unos kilómetros más allá. Y como se vio que la cosa no funcionaba, es decir que los nuevos equipamientos no generaban beneficios instantáneos, o se los mantuvo como auténticos espejismos grandilocuentes sin más sentido que su presencia fantasmal o, directamente, se los condenó al ostracismo. No se actuó en términos de identificación, memoria, sensibilización o educación, sino de planteamientos estricta y reductivamente economicistas.

Además, como el afán economicista guiaba, subrepticia o explícitamente, la escenificación general, se recurrió -donde se pudo - a la espectacularidad como reclamo visual y vivencial (Arrieta 2014). Con efectos paradójicos, una vez más: a veces la puesta en escena se convertía en más importante que los propios acervos escenificados -si es que existían, porque se inauguraron muchas cosas carentes de contenidos- haciendo bueno aquello que decían McLuhan y Fiore (1992) de que el medio era no ya el mensaje sino el masaje.

De aquellos polvos, estos lodos. Polvos veloces, además, pues mientras se agitaban en turbulentos remolinos apenas si dejaban tiempo para la reflexión serena y ponderada, al extender y afianzar una acuciante necesidad de acción y de cambio: lo importante parecía ser el hacer algo, no cómo se hacía, ni por qué, ni para qué ni para quién. Y lodos espesos. Confusos. Propios de momentos de incertidumbre, de crisis. Una crisis global, antropológica, que nos dice a las claras que, en el campo que nos ocupa, el futuro de tanto equipamiento es muy poco halagüeño. A no ser que se cambien los modelos y se hagan las cosas de otra manera.

\section{Caminante, no hay camino, se hace camino al andar}

En esa fiebre natalista, o creacionista, de tanto nuevo equipamiento hubo de todo. Algunos nacieron modestos $\mathrm{y}$, aun pudiendo tener su origen 
en la citada catarsis patrimonializadora, pueden ser -en mayor medida que las grandes instituciones- el escenario de procesos de identificación de determinados colectivos sociales con sus acervos, con su memoria, con su pasado y su presente. De manera lenta pero constante, desde aquellos imponentes edificios de inspiración clásica que en el siglo XIX encarnaban las glorias del estado-nación, se ha pasado a construcciones más humildes -cuando las hay-, más mimetizadas con su entorno, que pueden albergar obras señeras, pero también testimonios singulares no tanto por su morfología o factura sino por su capacidad para ilustrar sobre procesos al margen de los habituales relatos de la Gran Historia, cuando no olvidados por la Historia (Clifford 1991: 225-6).

Quizá sea una consecuencia de los procesos de globalización en los que estamos inmersos: al fin y al cabo el patrimonio es de las pocas cosas en las que distintas colectividades se pueden diferenciar de otras próximas o más lejanas. Es decir, constituye -o puede constituir- un rasgo de identidad particularizada frente a la tendencia homogeneizadora, en todos los órdenes, de los tiempos actuales. Es un rasgo, además, sobre el cual actúan las mecánicas proyectivas de los individuos, estableciendo elementos de recognoscibilidad, identificación y sentido que permiten considerarlo como algo propio.

Las dinámicas tradicionales de patrimonialización apenas si han tenido en cuenta ese matiz. $\mathrm{O}$ en todo caso lo han tratado a una escala que desvirtúa su potencial. En el momento que a un objeto se le asigna valor patrimonial, se establece en torno a él una serie de medidas que alteran lo que habría sido su vida "natural": registro, protección, reubicación -en muchos casos-, limitaciones en cuanto a su accesibilidad, etc. Es decir, se le proporciona una especie de corsé protector que, a su vez, implica un alejamiento de su contexto original -físico, social o conceptual-, insertándolo en un nuevo contexto donde se desarrollan nuevas dinámicas encomendadas a distintos especialistas. Estos actuarán con criterios profesionales, reelaborarán -o reeditarán- el producto y lo pondrán en circulación respetando principios racionales, objetivos y científicos pertinentes, pero incidirán poco o muy poco en aspectos de carácter emotivo, subjetivo o relacional (Schouten 1998).

Se institucionaliza esa elección y esa construcción patrimonial, aunque el proceso, claro está, pueda ser cuestionado tanto en sus orígenes como en sus consecuencias, como lo hace en forma de "pecio" Rafael Sánchez Ferlosio (2002: 112 y 115):

[...] me importa aquí la general brutalidad totalizadora (y al fin totalitaria) de los conceptos de Cultura y Patrimonio Cultural. La repugnante figura de Patrimonio Cultural es una exudación del 'ontologismo histórico' -creador de fetiches tales como 'El Ser de España'-, o sea cruda impostura e imposición dictada: 'Esta es tu herencia histórica, este es tu ADN cultural, esta es tu inalienable identidad'. La Cultura, instrumento de control social, induce a un halagador acatamiento. Ahora adopta el modelo del mercado y la publicidad [...] Aniquilado todo atisbo de sociedad civil, no queda ya más que el Estado como único posible gestor o sucedáneo, siquiera sea mecánico e indirecto, de la socialidad.

Si en su momento determinado bien era susceptible de ser investido de resortes emocionales -fervor religioso, en el caso de una imagen piadosa; placer de la posesión en el caso de una colección privada; sesgo emblemático de un bien colectivo, etc.- por parte de sus usuarios, en adelante estos deberán someterse al filtro de lo racional, de lo institucionalmente instaurado como patrimonio, hasta el punto de que, al cabo de los años, aquellos valores emotivos desaparecerán casi por completo: de alguna manera el bien se fosiliza; su integridad física queda a salvo, pero se desvanece el soplo vital que los alentaba. ¿Quién se emociona -en clave, por ejemplo, de nostalgia positiva o negativa- ante un artefacto de cualquier museo de etnografía? En todo caso, quien sabe para qué servía, cómo se usaba y qué implicaciones tenía en la vida cotidiana de quienes lo utilizaban, máxime si tal objeto le había afectado personalmente. Algo, se dirá, que evidentemente podrá ser explicado mediante un texto de apoyo. Sí, pero la percepción del nuevo usuario del bien, del visitante "lego", estará basada en lo racional, en lo pretendidamente científico, base argumentativa del dictamen del ente autorizado para decidir lo que es patrimonio, lo que ha de conservarse y lo que ha de exhibirse -opciones políticas, recordemos-, por lo que difícilmente implicará la misma carga de emotividad percibida por el conocedor, carga que es la que precisamente otorga todo su sentido al objeto en cuestión (Csikszentmihalyi \& Rochberg-Halton 1981). 
A un museo se acude, excepto en el caso de profesionales, estudiantes, escolares y otros sufridores de lo que podríamos llamar "imperativo categórico museológico" -los públicos cautivos aun en contra de su voluntad-, de manera voluntaria y planificada de acuerdo con las posibilidades de tiempo libre. Si hiciéramos un repaso a cuáles son las actividades más habituales y placenteras para ocupar ese tiempo de ocio, aunque resulten onerosas para nuestros bolsillos, tendríamos que citar, entre otros, la práctica de -o la asistencia como espectador a- actividades deportivas, la visita a parques temáticos, los viajes, las actividades gastronómicas, la frecuentación de tiendas, etc. Si nos fijamos un poco, todas ellas tienen que ver con la novedad, con la sorpresa, con lo sensorial, con la emoción -incluso con la pasión-, con la secreción de adrenalina -o, al contrario, con el relax más absoluto-, con la competencia y con la satisfacción.

Por su lado, Middleton (1985) llegaba hace varias décadas a la conclusión de que el objetivo último de quien acudía a un museo era salir satisfecho, además de haber vivido una experiencia al margen de la cotidianeidad, cargada de estímulos y generadora de entretenimiento y conocimiento. La visita -y más la frecuentación- de enclaves patrimoniales siempre -o en la mayoría de los casos- es una experiencia al margen de lo cotidiano. Y la experiencia tiene que ver con el placer -por lo general la gente no es masoquista y difícilmente estará dispuesta a emplear su tiempo libre en actividades poco gratificantes o desagradables-, con la socialización -el compartir experiencias satisface nuestra necesidad de comunicación, y de ahí que a esas visitas se acuda mayoritariamente en compañía (McManus 1987)-, con determinados comportamientos ritualizados -la moda del consumo cultural o los itinerarios jerárquicos que los visitantes dibujan en una suerte de mapa cognitivo al acudir a tal o cual enclave-, con la escenificación del acontecimiento -nada que ver las mortecinas salas de algunos museos con la parafernalia multimedia que despliegan otros- $y$, por no alargar el recuento, con el aprendizaje.

Nos podríamos preguntar, entonces, si todos esos factores se tienen en cuenta -y en qué orden de prelación- a la hora de emprender una activación patrimonial, y si realmente las estrategias están encaminadas a lograr la estimulación -y la satisfacción- de todos los vectores que convierten una experiencia en una experiencia gratificante. En las experiencias patrimoniales al uso hay, evidentemente, una ruptura con la cotidianei- dad, hay entretenimiento, hay estímulos y algo se puede aprender a poco que nos empeñemos en ello. Pero ¿cuánto hay de novedad o de sorpresa al margen de las exposiciones temporales? ¿Por qué los estímulos sensoriales se reducen a lo puramente visual en la mayoría de los casos? ¿Cuánta emoción experimentamos y cuánta adrenalina secretamos? ¿O es que salimos relajados o, más bien, cansados, dada la habitual racanería de quienes se deberían encargar de hacernos la vida más feliz o, al menos, más cómoda, a la hora de disponer de elementos de descanso? ¿Nos sentimos competentes o incompetentes mientras deambulamos entre obras explicadas en un lenguaje o muy banal o muy especializado? ¿Y acaso no nos sentimos más competentes en las tiendas y en la cafetería del museo? ¿Salimos realmente satisfechos de nuestra experiencia? $\mathrm{Si}$ sí ¿por qué, y volvemos al principio, tan poca gente acude a los enclaves patrimoniales?
$\mathrm{Al}$ andar se hace el camino,
$\mathrm{Y}$ al volver la vista atrás
Se ve la senda que nunca
se ha de volver a pisar.

Los procesos de activación patrimonial al uso introducen un vector de artificiosidad y una jerarquización en los roles desempeñados por los distintos protagonistas de sus mecánicas. Por un lado, al rodear al bien de esa especie de envolvente protector, se lo está descontextualizando -y, paralelamente, contextualizándolo en otros parámetros-, con lo que se pierden nexos de unión con su primitivo contexto, necesarios para su cabal entendimiento y disfrute. Por otro, se establece una división jerárquica entre quienes tienen la potestad de decidir sobre las estrategias a seguir y quienes solo pueden acceder al bien siguiendo las pautas normativas de los primeros. A los teóricos propietarios del bien se les despoja de la posibilidad de decisión sobre ellos, haciendo buena la afirmación de Carol Duncan (1995: 8-9) cuando decía que controlar el museo -o el patrimonioimplicaba controlar la representación de los más altos valores y verdades de una comunidad: quien controle esa representación estará, a su vez, definiendo quién(es) constituye(n) la comunidad y cómo se define su identidad.

Una vez que la activación patrimonial ha tomado cuerpo, el resultado es un discurso las más de las veces cerrado, inamovible durante largo tiempo, hasta que alguien, investido de la potestad de hacerlo, lo corrija, lo cambie o lo plasme 
en otros formatos acordes con los nuevos tiempos o con las posibilidades técnicas, siempre cambiantes, siempre en evolución. Pero ese alguien siempre pertenecerá a los niveles superiores de la jerarquía, casi nunca provendrá de la mayoría de agentes pasivos, condenados a la condición de comparsas, de públicos, de visitantes de las mecánicas patrimoniales. El patrimonio se transmuta en un resorte de poder, y este impone su discurso, su modelo de interpretación sobre algo que, en principio, pertenece a todos.

Desde finales de la II Guerra Mundial se empezaron a oír voces que reclamaban un protagonismo creciente para el teórico destinatario de los desvelos patrimoniales. Al fin y al cabo, el patrimonio no tiene sentido per se, sino en tanto en cuanto resulta operativo para algo al servicio de alguien. Desde las proclamas y modos de hacer de las Nuevas Museologías hasta la proliferación de actividades didácticas son respuestas, cada una de naturaleza e incidencia específicas, a esa reivindicación asumida mayoritariamente en términos generales, pero que no acaba de romper con unas inercias y unos modos de hacer propios de aquellos museos y enclaves patrimoniales como imagen y representación de los estadosnación decimonónicos. En el fondo, de lo que se trata es de redefinir el "alguien" a cuyo servicio debe estar el patrimonio.

Si al "sujeto" -individual y/o colectivo- se le margina en las tomas de decisión realmente importantes, se impone un alejamiento entre dicho sujeto y el bien. Por muy bien conservado y exhibido que esté, ese alejamiento reforzará el desvanecimiento de valores emotivos y proyectivos para un sector mayoritario de la población y solo cobrará sentido para una minoría: los conocedores, especialistas, coleccionistas, etc. Al resto, a la mayoría, se tratará con abundantes dosis de indulgencia y paternalismo, que no constituyen sino otras tantas caras de una concepción autoritaria del patrimonio: los discursos no se negocian, se imponen; y para suavizar el trago, se ofertan cápsulas informativas o de acción que, al margen de su utilidad, recuerdan a sus beneficiarios su condición de parias, de legos, de ignorantes en asuntos patrimoniales.

Eso si en aras de la conservación el bien no ha sido condenado a las insondables tinieblas de los almacenes, lugares sacrosantos e inaccesibles pronto saturados por un incesante flujo de obras que obligará, tarde o temprano, a replantearse el espinoso asunto de la ética de la conservación, solventado por lo general por esa especie de cantinela que afirma que una de las funciones de museos y centros patrimoniales es aumentar sus colecciones y que acabará por llevarlos a auténticos callejones sin salida. Al sujeto no le quedará otra opción que entrar en el juego con todas sus consecuencias. Acudirá al enclave patrimonial, lo que denota elevación de miras y sensibilidad -distinción, que diría Bourdieu (1988)-. Entenderá más o menos lo que se le cuenta, pero procurará que sus lagunas informativas -o su ignorancia- no resulten demasiado evidentes. Saldrá más o menos satisfecho de la experiencia, pero nunca confesará abiertamente que se ha aburrido o que no ha entendido. Y, en todo caso, observará con cierta distancia atemperadora aquellas partes del discurso que no casan con sus convicciones, pues sabe -y lo acepta- que en ningún caso podrá alterar la propuesta que se le ofrece al no estar investido de potestad para hacerlo. Finalmente, mariposeará y comprará en la tienda -poblada de objetos de "diseño", lo que también es de buen tono- $y$, con un poco de suerte, se llevará a casa un catálogo o un póster de la exposición, lo que certificará su pertenencia -en mayor o menor grado- al exquisito universo de las élites que practican el consumo cultural.

Desde hace tiempo el visitante presta más atención a lo colateral -tiendas, bares, restaurantes, reproducciones, librería- que a lo que en principio debería ser el epicentro del museo o centro patrimonial -las obras, las exposiciones, las actividades- (Perniola 1989: 151). Cabría, por tanto, preguntarse a qué responde este comportamiento, por qué el visitante se siente más cómodo en unos lugares que en otros, y precisamente en esos. Porque, en principio, los poderes públicos no ponen en marcha activaciones patrimoniales para invitar al consumo a sus ciudadanos... ¿o sí?

Como la visita cansa física y psicológicamente, tanto por los ritmos de desplazamiento no habituales como por la atención que se nos exige para acceder al entendimiento de los discursos, y como además el visitante se ubica en la base de la pirámide, la que corresponde a quienes no son del todo competentes, el recurso a los lugares colaterales que mencionaba Perniola es una de las salidas lógicas a fin de lograr ciertas dosis de equilibrio y satisfacción. Se acude al bar, se visita la tienda, colocada estratégicamente para que constituya un paso obligado -y en menor medida la librería, si existe-, y se da rienda suelta al cúmulo de emociones anestesiadas en la visita propiamente dicha, satisfaciendo pulsiones viscerales relacionadas con el consumo y moviéndose 
con la seguridad que otorgan la competencia que había sido negada ante los bienes patrimoniales, el deliquio de la posesión y la experimentación de placeres sensoriales sin cortapisas.

Reconducir este estado de cosas resulta difícil, sobre todo en enclaves patrimoniales que reciben un considerable número de visitas. Máxime si nos movemos en unos vectores caracterizados por patrones de consumo y mecánicas de delegación, esas que otorgan a los poderes públicos la potestad de gestionar sus bienes, conformándose el otorgante con el disfrute de los productos elaborados por dichos poderes, sin que existan canales realmente eficientes para intervenir en la toma de decisiones: si eso pasa en la economía o en la política, y pasa, y de qué manera, cómo evitarlo en el campo patrimonial.

Aun así no estaría de más intentar otras vías, que podrían resultar más efectivas en esos equipamientos más modestos que durante años se han movido en clave de mímesis respecto a los modelos de referencia, en pequeñas o medianas instituciones que pueden recuperar, mediante mecánicas no solo racionales sino también emotivas y proyectivas, los nexos de unión entre sujetos y acervos patrimoniales. Para ello, evidentemente, habría que empezar por pensar en el patrimonio desde una óptica diferente y trabajar siguiendo unos principios inclusivos que intenten aunar todas las potencialidades de unos y otros.

El patrimonio lo construimos entre todos, luego todos deberíamos ser agentes activos en las mecánicas que en torno a él se puedan articular, y romper con las falsas bipolaridades que atribuyen a unos la potestad de acción y a otros los confinan a desempeñar papeles pasivos. Ello no quiere decir que los poderes públicos no puedan intervenir motu proprio: solo hay que recordar los casos de bienes patrimoniales en serio riesgo de desaparición por el desconocimiento, la insensibilidad o la desidia generalizados (véase un ejemplo periodístico en Verdú 2014). Ahí sí, la labor de los especialistas capaces de definir campos de actuación, establecer prioridades y articular estrategias resulta indispensable a fin de lograr una viabilidad efectiva en el mantenimiento del bien, bien por actuaciones directas de las administraciones -difícil en los tiempos de penuria que corren-, bien mediante fórmulas que involucren a la iniciativa privada.

Ahora bien, las dinámicas de delegación a que estamos acostumbrados dificultan la pervivencia de algo que antaño era indispensable y se practicaba con regularidad: el trabajo comunal. Muchos ya ni siquiera saben qué es eso. Y, sin embargo, constituye una de las vías más efectivas para que el común de los mortales se involucre en tareas patrimoniales y se configure como un interlocutor indispensable ante expertos o administraciones. Podría parecer una quimera, tal como están las cosas, pedirle a alguien que participe de modo altruista en un grupo de trabajo a la hora de gestionar una actividad patrimonial: eso significa esfuerzo, tiempo, generosidad, etc. De todas formas, el movimiento asociacionista goza de buena salud: una magnífica red donde buscar complicidades siempre que el esfuerzo tenga su recompensa.

Una recompensa que puede materializarse de distinta manera: la posibilidad de interactuar con otras personas para alcanzar un logro común, la comprobación de que el esfuerzo altruista puede servir para obtener beneficios -no necesariamente materiales-, aumentar el nivel de bienestar personal y social o ejercer cotas de poder o, al menos, ejercer cierta influencia sobre el entorno (Sánchez Santa-Bárabara y García Martínez 2001: 174). No se trata pues de pedir colaboración a los colectivos organizados. Se trata de devolverles la potestad de intervenir en la activación patrimonial desde su inicio, de manera que su impronta se vea reflejada en el resultado final y no constituya solo la plasmación de una idea enunciada por la institución y filtrada, parcheada o corregida por el grupo de voluntarios.

Actuar así implica cambios radicales en las habituales mecánicas patrimoniales. Implica trabajar en un sistema en el cual la interlocución se instaura como eje nuclear de cualquier actividad. Implica conocer el patrimonio, pero también conocer la vinculación -real y posible- de los colectivos sociales con ese patrimonio y los beneficios que de esa relación se pueden derivar. Implica erradicar el dirigismo y la separación entre agentes activos y pasivos. Implica huir de la sacralización del pasado y utilizar el patrimonio como herramienta que incida en el presente y en sus desvelos y preocupaciones. Implica huir del economicismo subyacente a la búsqueda indiscriminada del turismo como destinatario -cuya relación con el patrimonio siempre será eventual, aleatoria y superficial-, para apostar por el sujeto natural, el de proximidad, aquel que lo puede investir de matices emotivos y en el cual puede proyectar toda suerte de valores, más allá de los puramente racionales o científicos. Implica todo eso y más, pero sobre todo implica un cambio radical en el funcionamiento de nuestras sinapsis cerebrales cuando se activan en relación al patrimonio. 


\section{REFERENCIAS Bibliográficas}

Arrieta URTizBerea, I. (2012): La interrelación entre los sistemas turístico y patrimonial: más allá de los discursos apologéticos y las prácticas reduccionistas. Museo y turismo: expectativas y realidades (I. Arrieta, ed.), UPV/EHU, Bilbao: 11-24.

Arrieta Urtizberea, I. (2014): Museos en la posmodernidad: retos y desafíos. Reinventando los museos, UPV/EHU, Bilbao: 11-26.

Bourdieu, P. (1988): La distinción. Criterios y bases sociales del gusto, Taurus, Madrid.

Clifford, J. (1985): Objects and Selves - An Afterword. Objects and Others: Essays on Museums and Material Culture (G.W. Stocking Jr., ed.), History of Anthropology, vol. 3, University of Wisconsin Press. Madison: 236-246.

Clifford, J. (1991): Four Northwest Coast Museums: Travel Reflections. Exhibiting Cultures. The Poetics and Politics of Museum Display (I. Karp, S. D. Lavine, eds.), Smithsonian Institution Press, Washington and London: 212-254.

Csikszentmihalyi, M.; Rochberg-Halton, E. (1981): The Meaning of Things, Cambridge University Press, London.

Duncan, C. (1995): Civilizing Rituals: Inside Public Art Museums, Routledge, London and New York.

Donaire, J.A. (2012): Turistas y museos. Apocalípticos e integrados. Museos y turismo: expectativas y realidades (I. Arrieta, ed.), UPV/EHU, Bilbao: 25-38.

Hudson, K. (1989): Un museo innecesario. Museum, 162: 114-116.

Karo, I. (1991): Culture and Representation. Exhibiting Cultures. The Poetics and Politics of Museum Display (I. Karp, S.D. Lavine, eds.), Smithsonian Institution Press. Washington and London: 11-24.

McLuhan, M. \& Q. Fiore (1992): El medio es el masaje, Paidós Ibérica, Barcelona.

McManus, P. (1987): Communication With and Between Visitors to a Science Museum, London University, London.

Middleton, V. T.C. (1985): Museums Are for People, Scottish Museums Council, Edinburgh.

Perniola, M. (1989): Museos y colecciones. Revista de Occidente, no 100: 142-152.

Prats, Ll. (2003): Patrimonio + turismo = ¿desarrollo? Pasos, vol. 1, nº 2: 127-136.

SÁnchez Ferlosio, R. (2002): La hija de la guerra y la madre de la patria, Destino, Barcelona.

Sánchez Santa-Bárbara, E.; García Martínez, M. A. (2001): Análisis de las motivaciones para la participación en la comunidad. Papers, 63/64: 171-189.

Schouten, F. (1998): Profesionales y público: un acercamiento necesario. Museum, no 200: 27-30.

Verdú, D. (2014): Catedrales de una religión industrial. El País, 30 de marzo: 48. 